\title{
Study on Lubrication Efficiency and Friction Power Loss of Engine Based on a Hybrid Hydrodynamic Model
}

\author{
R.Q. Jiao ${ }^{1,2}$ and V.L. Nguyen $1,2,3,{ }^{*}$ \\ ${ }^{1}$ School of Mechanical and Electrical Engineering, Hubei Polytechnic University, 435003 Huangshi, China \\ ${ }^{2}$ Hubei Key Laboratory of Intelligent Conveying Technology and Device, Hubei Polytechnic University, 435003 Huangshi, China \\ ${ }^{3}$ Faculty of Automotive and Power Machinery Engineering, Thai Nguyen University of Technology, 24000 Thai Nguyen, Vietnam
}

\begin{abstract}
Friction loss is one of the main factors affecting engine power. Reducing friction power losses to improve the power of engines is a significant concern for designers. Especially, under the background of energy-saving and emission reduction, it is indispensable to carry out an in-depth investigation on engine bearing lubrication characteristics. Unlike the previous studies of separate modelling, a new modelling method of coupling the dynamic and lubrication model is proposed in this paper. The bearing capacity, friction force, friction coefficient and eccentricity ratio were taken as the evaluation criterion, and the influence of design parameters such as angular speed, bearing radius and width on the lubrication efficiency and friction power loss (LE-FPL) were studied. The results indicate that increasing the angular speed, bearing radius or width can effectively reduce the eccentricity ratio and raise the minimum oil film thickness, which is beneficial to improve the lubrication efficiency. However, the above methods to improve engine lubrication efficiency will lead to more power loss of engine to a certain extent. Therefore, studies on reducing the friction power loss for the engine and on improving the lubrication efficiency for the engine should be considered coordinately in the dynamic design and optimisation of the engine.
\end{abstract}

ARTICLE HISTORY

Received: $18^{\text {th }}$ Aug 2020

Revised: $26^{\text {th }}$ May 2021

Accepted: 21 $1^{\text {st }}$ Aug 2021

\section{KEYWORDS}

Crankpin bearing;

Lubrication efficiency;

Friction power loss;

Slider-crank-mechanism

\section{INTRODUCTION}

With the continuous improvement of people's requirements for fuel economy and energy conservation and emission reduction for the oil-fueled automotive, reducing friction power losses to enhance engine power has been a significant concern for automotive designers. It turns out that the main reason for engine power loss is the friction between the piston skirt and the piston ring on the cylinder bore during the motion[1-2]; and the friction of the relative moving surface pairs of the joints of the slider-crank-mechanism (SCM) [3]. Over the last decades, the effect of design parameters of the SCM, the piston's inertial mass, and eccentricity between crankshaft centre and cylinder centre on the horizontal impact force of the piston on the cylinder were investigated [4-6]. It found that the friction and noise vibration generated by the piston skirt and ring against the cylinder bore substantially decreased with the optimised SCM's structure. Furthermore, for optimisation, the lubrication factors, including friction force and friction coefficient between relative moving surface pairs of the piston-cylinder, had also been investigated $[1-2,6]$. They found that when the thickness of oil film on the contact surface remains stable, the friction power losses of the SCM substantially decreased. However, the oil film thickness is affected not only by the speed and load of the SCM but also by its thermal and dynamic viscosity. Research on the characteristics and optimisation of the heat transfer efficiency of the oil were also performed [7-9], and the neuro-network method was then applied to predict the dynamic viscosity of the oil during the working period [10]. Therefore, the film thickness of the oil could control.

Besides, to illustrate the stability of the oil film thickness of the friction surface pairs in an engine, especially the crankpin bearing (CB), a CB's lubrication model was built under different conditions of external load and speed of the shaft; based on the evaluation indexes of the friction force and load-bearing capacity [11-13]. The results indicated that the stability of oil film thickness strongly depended on the oil film pressure. In addition, the effects of some factors such as the temperature [14], the radial clearance [15-16] and the load of the shaft $[12-13,17]$ on the oil film pressure were also discussed. However, in the above researches, the CB's lubrication efficiency is mainly evaluated under a condition of static load at the high speed of the shaft. In actual conditions, the generated shear stress of the oil film thickness could also influence the CB's resistance under the change of the CB's speeds, loads, and dimensions, respectively. Furthermore, during a complete cycle of the engine, the dynamic load on $\mathrm{CB}$ varies with the angular velocity. Thus, it is necessary to take the effects of the angular speeds and dimensions of the CB into account during the analysis of the lubrication efficiency and friction power loss (LE-FPL) for the engine. However, the existing investigations on the LE-FPL for engines considering the effect of the angular speeds and the dimensions of the $\mathrm{CB}$ together were rarely reported.

Given the above, this paper is focused on establishing a new hybrid, an SCM dynamics model and the CB lubrication model, to further investigate the influence of angular speeds and dimensions of the CB on LE-FPL for engines by the method of co-simulation. Based on the hybrid model of the SCM and CB, an algorithm program written in MATLAB was performed to solve the hydrodynamic equations of the SCM and CB. The load-bearing capacity, friction coefficient, 
friction force, and eccentricity ratio between the shaft and bearing were selected as the objective functions. Then the effect of the different angular speeds, bearing radii and widths of the CB on the LE-FPL were evaluated. The new contributions of this study lie in the following aspects: (i) a new numerical method established by combining the SCM dynamic model and the CB lubrication model to investigate the LE-FPL of engines has been proposed, (ii) the influence of the eccentricity between the shaft and the bearing on vibration and lubrication stability has also been revealed and, (iii) both lubrication efficiency and friction power loss of the engine under different angular speeds, loads, and dimensions of the CB have been analysed.

\section{HYBRID DYNAMICS AND LUBRICATION MODELLING}

\section{Dynamic Model of Slider-Crank-Mechanism}

Figure 1 presents the SCM dynamic model and the symbols list in Table 1. According to the diagram, the motion equation of the piston can be expressed as

$$
z=l(1-\cos \delta)+r(1-\cos \phi)
$$

Assume $\lambda=r / l$ represents the ratio of rotation radius of the crankshaft to the length of connecting rod, so the kinematic relation of SCM can be written as:

$$
\left\{\begin{array}{l}
\cos \delta=\sqrt{1-\lambda^{2} \sin ^{2} \phi} \approx 1-0.5 \lambda^{2} \sin ^{2} \phi \\
\sin \delta=\lambda \sin \phi
\end{array}\right.
$$

Combining Eq. (1) and (2), the motion equation of the piston can be deformed into:

$$
z=r\left(1+\frac{\lambda}{4}-\cos \phi-\frac{\lambda}{4} \cos 2 \phi\right)
$$

Then, the piston acceleration response can be calculated by:

$$
\ddot{z}=\frac{d z^{2}}{d t^{2}}=\omega^{2} r(\cos \phi+\lambda \cos 2 \phi)
$$

where $\omega=\dot{\phi}=d \phi / d t$

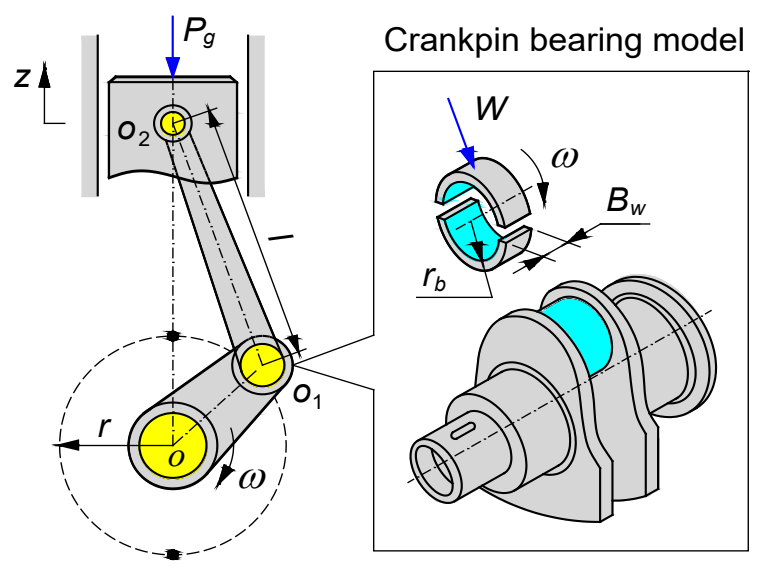

(a)

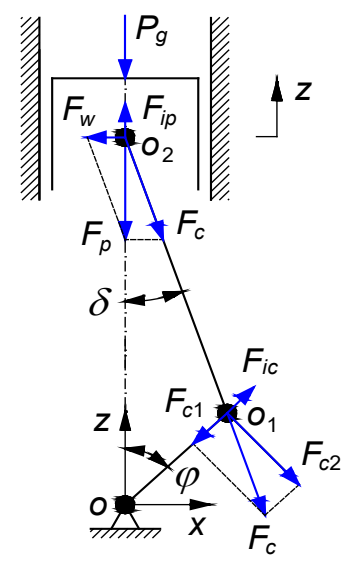

\begin{tabular}{|c|c|c|}
\hline & Nomenclature & Nomenclature \\
\hline$l$ & connecting rod length/ $\mathrm{m}$ & inertial force of the small-rod-end/ $\mathrm{N}$ \\
\hline$r$ & rotation radius of the crankshaft/ $\mathrm{m}$ & piston force impacting on the connecting rod/ $\mathrm{N}$ \\
\hline$P_{g}$ & combustion gas pressure/ $\mathrm{kPa}$ & the total force of the piston/ $\mathrm{N}$ \\
\hline$r_{b}$ & crankpin bearing radius/ $\mathrm{m}$ & connecting rod angle/ deg \\
\hline$\omega$ & the angular speed of the crankshaft/ $\mathrm{rad} / \mathrm{s}$ & radial force/ $\mathrm{N}$ \\
\hline$\varphi$ & the crankshaft angle/ deg & tangent force/ $\mathrm{N}$ \\
\hline$B_{w}$ & crankpin bearing width/m & centrifugal inertial force of the large-rod-end/ $\mathrm{N}$ \\
\hline$W$ & impact force on the crankpin bearing/ $\mathrm{N}$ & piston force impacting on the cylinder wall/ $\mathrm{N}$ \\
\hline
\end{tabular}

(b)

Figure 1. The model of (a) lubrication joint of crankpin bearing and (b) SCM dynamics

Table 1. The symbols of the S.C.M dynamics model 
It assumed that $m_{b}$ and $m_{s}$ are the lumped mass of the big- and small-rod-end of the connecting rod at $o_{1}$ and $o_{2}$, and the piston mass represented by $m_{p}$, so the inertial force of the small-rod-end and piston can be determined by $m_{\mathrm{p}}$. Thus, the inertial force of the small-rod-end and piston can be determined by:

$$
F_{i p}=-\left(m_{p}+m_{s}\right) \omega^{2} r(\cos \phi+\lambda \cos 2 \phi)
$$

When the piston moves to its apex, the impact force $P_{g}$ produced by the cylinder wall and the connecting rod can be written as:

$$
\left\{\begin{array}{l}
F_{c}=\left(P_{g}+F_{i p}\right) / \cos \delta \\
F_{w}=\left(P_{g}+F_{i p}\right) \tan \delta
\end{array}\right.
$$

In addition, the two components of tangential and radial forces of impact of $F_{c}$ on the $\mathrm{CB}$ are given by

$$
\left\{\begin{array}{l}
F_{c 1}=F_{c} \cos (\phi+\delta) \\
F_{c 2}=F_{c} \sin (\phi+\delta)
\end{array}\right.
$$

Considering the effect of angular speed $\omega$, the centrifugal inertial force of the big end of connecting rod can be written as:

$$
F_{i c}=-m_{b} r \omega^{2}
$$

The primary function of connecting rod bearings is to provide rotary motion and transfer load, and the dynamic load $W$ of the connecting rod impacted on the bearing of the crankpin can be defined as:

$$
W=\sqrt{\left(F_{c 1}+F_{i c}\right)^{2}+F_{c 2}^{2}}
$$

Then the LE-FPL characteristics of the CB were studied with the change of $W$ in direction and strength.

\section{Crankpin Bearing Lubrication Modelling}

\section{Hydrodynamics lubrication model}

The structure model and lubrication model of CB are shown in Figure 2(a) and Figure 2(b), respectively. When the engine is working, the crankpin is affected by the dynamic load $W$ and rotates in the connecting rod bearing at an angular speed $\omega$. Herein, the symbols in Figure 2 are listed in Table 2.

\section{Application of the Reynolds equations}

Assuming that the bearing surface is fixed in both $x$ - and $y$-directions, and the shaft only moves with a constant speed $u$ in the $x$-direction, shown in Figure 2. Thus, the boundary conditions of the fluid on the CB surfaces in the $x$ - and $y$ directions are defined as:

$$
\left\{\begin{array}{l}
U_{(z=0)}=0, \text { on bearing surface } \\
U_{\left(z=h_{f}\right)}=u, \text { on crankpin surface }\{
\end{array}\right.
$$

Also, assuming that the physical parameters of the lubricating oil, such as density and viscosity, are constant without being influenced by other factors, and the influence of the flow inertia of the lubricating oil during the working process can neglect. Hence, oil film pressure distribution can be determined by $[13,16]$

$$
\left\{\begin{array}{l}
\frac{\partial p}{\partial x}=\frac{\partial}{\partial z}\left(\eta \frac{\partial U}{\partial z}\right) \\
\frac{\partial p}{\partial y}==\frac{\partial}{\partial z}\left(\eta \frac{\partial V}{\partial z}\right)
\end{array}\right.
$$

Combining Eq. (10) and Eq. (11), and marking the dynamic viscosity of oil film with $\eta$, the fluid velocity equations can be obtained through integral operation as shown below.

$$
\left\{\begin{array}{l}
U=\frac{1}{2 \eta} \frac{\partial p}{\partial x}\left(z^{2}-h_{f} z\right)+u \frac{z}{h_{f}} \\
V=\frac{1}{2 \eta} \frac{\partial p}{\partial y}\left(z^{2}-h_{f} z\right)
\end{array}\right.
$$




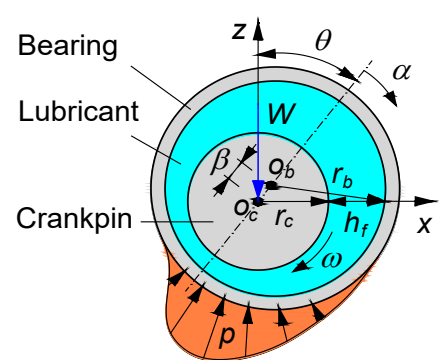

(a)

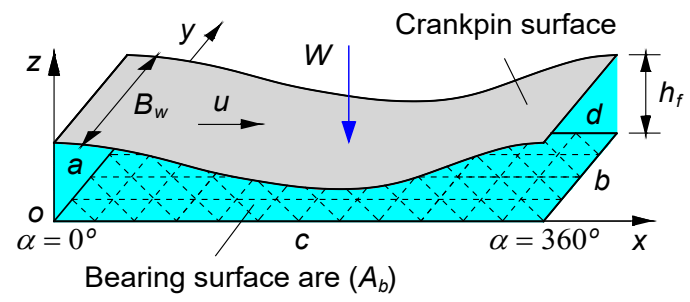

(b)

Figure 2. The CB hydrodynamics model.

Table 2. The symbols of the CB hydrodynamic model.

\begin{tabular}{|c|c|c|c|}
\hline \multicolumn{4}{|c|}{ Nomenclature } \\
\hline$A_{b}$ & Bearing surface area $/ \mathrm{m}^{2}$ & $p$ & hydrodynamic pressure of the oil film/ $\mathrm{Pa}$ \\
\hline$a, b$ & $\begin{array}{c}\text { boundary lines of the initial and final pressures at } \\
\text { the maximum } h_{f}\end{array}$ & $u$ & $\begin{array}{l}\text { the relative velocity between the CB's } \\
\text { surfaces }\left(u=r_{b} \omega\right) / \mathrm{m} / \mathrm{s}\end{array}$ \\
\hline$c, d$ & $\begin{array}{l}\text { boundary lines of the right and left pressures of the } \\
\text { bearing at } y=0 \text { and } B_{w}\end{array}$ & $\gamma$ & $\begin{array}{l}\text { eccentricity ratio between centre of bearing } \\
\text { and shaft }(\gamma=\beta / \kappa \text {, and } 0<\gamma<1)\end{array}$ \\
\hline$B_{w}$ & bearing width $/ \mathrm{m}$ & $\kappa$ & $\begin{array}{l}\text { radial clearance of shaft and bearing }\left(\kappa=r_{b}-\right. \\
\left.\qquad r_{c}\right) / \mu \mathrm{m}\end{array}$ \\
\hline$r_{b}$ & bearing radius/ $\mathrm{m}$ & $W$ & dynamic load of the $\mathrm{CB} / \mathrm{N}$ \\
\hline$r_{c}$ & crankpin radius $\left(r_{c}<r_{b}\right) / \mathrm{m}$ & $\omega$ & angular velocity of the crankpin/ $\mathrm{rad} / \mathrm{s}$ \\
\hline$\beta$ & eccentricity centre of bearing and shaft/ $\mathrm{m}$ & $o_{b}, o_{c}$ & center of bearing and crankpin \\
\hline$\alpha$ & angular coordinate $\left(0^{\circ} \leq \alpha \leq 360^{\circ}\right) / \operatorname{deg}$ & $h_{f}$ & $\begin{array}{l}\text { the oil film thickness of } \mathrm{CB}\left(h_{f}=\kappa(1+\gamma\right. \\
\qquad \operatorname{Cos} \alpha) / \mu \mathrm{m}\end{array}$ \\
\hline$\theta$ & attitude angle/ deg & $x, y, z$ & coordinate axes \\
\hline
\end{tabular}

Integrating Eq. (12) in the z-direction, we can obtain the volumetric flow in the x- and y-direction, marked as $Q_{x}$ and $Q_{y}$, respectively.

$$
\left\{\begin{array}{l}
Q_{x}=\int_{0}^{h_{f}} U d z=-\frac{h_{f}^{3}}{12 \eta} \frac{\partial p}{\partial x}+u \frac{h_{f}}{2} \\
Q_{y}=\int_{0}^{h_{f}} V d z=-\frac{h_{f}^{3}}{12 \eta} \frac{\partial p}{\partial y}
\end{array}\right.
$$

Here, the fluid continuity condition is given in the form as:

$$
\left[\frac{\partial(\rho U)}{\partial x}+\frac{\partial(\rho V)}{\partial y}+\frac{\partial(\rho \varpi)}{\partial z}\right]+\frac{\partial \rho}{\partial t}=0
$$

Where $\rho$ is fluid density, $U$ and $V$ represent the fluid velocities in $x$ - and $y$-directions determined by Equation (12), and $\varpi$ represents the fluid velocity in the $z$-direction.

Neglect of the small quantity $\varpi$ and assume that $\rho$ is constant. By integrating Eq. (13) in the z-direction, we have:

$$
\frac{\partial}{\partial x}\left(\int_{0}^{h_{f}} U d z\right)+\frac{\partial}{\partial y}\left(\int_{0}^{h_{f}} V d z\right)+\frac{\partial}{\partial t}\left(\int_{0}^{h_{f}} d z\right)=0
$$

Due to the oil film thickness $h_{f}=\kappa(1+\gamma \cos \alpha)$ varying with the rotation angle $\alpha$, thus, combining Eq. (13), Eq. (15) can be rewritten as

$$
\frac{\partial}{\partial x}\left(h_{f}^{3} \frac{\partial p}{\partial x}\right)+\frac{\partial}{\partial y}\left(h_{f}^{3} \frac{\partial p}{\partial y}\right)=6 \eta u \frac{\partial h_{f}}{\partial x}+12 \eta \frac{\partial h_{f}}{\partial t}
$$

Based on the object of this study, the dimensionless form of the Reynolds equation of Eq. (16) is modified as:

$$
\chi^{2} \frac{\partial}{\partial \alpha}\left(H^{3} \frac{\partial P}{\partial \alpha}\right)+\frac{\partial}{\partial Y}\left(H^{3} \frac{\partial P}{\partial Y}\right)=\Delta \frac{\partial H}{\partial \alpha}+\Omega \frac{\partial H}{\partial T}
$$

where $\chi=B_{w} / r_{\mathrm{b}}, H=h_{f} / \kappa, \alpha=x / r_{\mathrm{b}}, Y=y / B_{w}, P=p / p_{0}, T=t / t_{0}, \Delta=6 \eta \omega B_{w}{ }^{2} \omega / \kappa^{2} p_{0}, \Omega=12 \eta B_{w}{ }^{2} / \kappa^{2} t_{0} p_{0}$, and $p_{0}=$ $101325(\mathrm{~Pa})$ is the standard atmospheric pressure. 
To obtain the pressure distribution of the oil film, the boundary conditions need to be determined first. In this study, we made the following assumptions: (i) the oil film thickness $h_{f}$ exists over CB surface, and (ii) the inlet and outlet lubricants are at the maximum position of $h_{f}$, and (iii) the ambient pressure around of the CB, inlet and outlet pressures equal the atmospheric pressure $p_{0}$. The computational region $A_{b}$ of the $\mathrm{CB}$ is also plotted in Figure 2(b).

Herein, the boundary lines of $a$ and $b$ at the maximum position of $h_{f}$ corresponding to $\alpha=0^{\circ}$ and $\alpha=360^{\circ}$ are defined as the initial and final pressures of the model, and the boundary lines of $c$ and $d$ at $y=0$ and $B_{w}$ are defined as the right and left pressures of the bearing. Hence, the oil film boundary conditions are defined by

$$
\left\{\begin{array}{l}
p_{(y=0)}=p_{\left(y=B_{w}\right)}=0 \\
p_{\left(\alpha=0^{\circ}\right)}=p_{\left(\alpha=360^{\circ}\right)}
\end{array}\right.
$$

Studies show that the oil film in the $\mathrm{CB}$ is usually divided into the liquid zone and cavitation zone, in which the oil film often ruptures [13]. Therefore, the cavitation region is often challenging to calculate effectively due to the negative pressure caused by gas dissolution and cavitation effects [18-19]. In this study, instead of ignoring negative pressures in the cavitation zone, only pressures below the saturation pressure, $p_{s}$ is varied to reach the effective pressure $p_{e}[15]$. Thus, the oil film pressure in the cavitation zone can be then expressed by $p_{e}=p_{s}$ if $p_{s}>p$ and $p_{e}=p$ if $p_{s} \leq p$.

\section{Lubrication Forces of the Mixed Hydrodynamics}

The load-bearing capacity (LBC) of the oil film pressure can be given as:

$$
W_{l}=\left[\left(-\iint_{A_{b}}^{\int} p \sin \alpha_{e}()^{2}\left(-\iint_{A_{b}}^{\int} p \cos \alpha_{e}()^{2}\right)\right)^{1 / 2}\right]
$$

Herein, the load-bearing capacity, $W_{l}$ equals the dynamic load $W$ of the connecting rod impacted on the CB. In region $A_{b}$, the friction force $F_{f}$ generated from the interfacial shear stress can be expressed as [20]:

$$
F_{f}=\iint_{A_{b}}^{\int}\left(\frac{h_{f}}{2} \frac{\partial p_{e}}{\partial x \frac{\eta}{h_{f}}}()\right)
$$

Once both unknowns of $W_{l}$ and $F_{f}$ obtained, the coefficient of friction of the CB (COF) can be calculated by:

$$
\text { C.O.F }=\frac{F_{f}}{W_{l}}
$$

\section{Evaluation Index and Simulation Algorithm}

Based on the MATLAB software, the system equations solution mainly includes the following steps.

\section{Set the initial parameters}

The initial parameters that need to be set include the dimension of the SCM and the CB, the combustion pressure on the piston peak, the zero matrices of the oil film pressure and shear stress $(n \times m)$ and the zero matrices of force vectors $(1 \times m)$. Then the dynamic load $W$ and coefficients of Eq. (17) were calculated to obtain the initial pressure and shear stress matrices. Herein, the number of grid nodes is $n=m=120$.

\section{Calculate the eccentricity ratio}

Theoretically, the load $W_{l}$ equals dynamic load $W$. However, the two are often not equal in practice. Thus, the computational algorithm cannot give the stopping condition for the computation process. In this study, a stop condition of $W-W_{l} \leq v$ with a minimal value $v$ was given. And when the stop condition is reached, the difference between $W_{l}$ and $W$ is tiny. The distribution of the oil film pressure and shear stress in the computational region and eccentricity ratio between journal and bearing are acceptable, and the computational algorithm stopped at this point. On the contrary, the computational algorithm continue until the stopping condition is satisfied.

\section{Calculate the loops}

The time taken for the engine to complete one cycle is $t_{0}$, which corresponds to the crankshaft rotation angle of $720^{\circ}$. Here, $t_{0}$ is divided into 120 equal parts, and $t$ is each time node corresponding to the crankshaft rotation angle of $t^{\prime}=6^{\circ}$. At each time $t$, the oil film pressure and shear stress were calculated, and then the computational algorithm continued for the next loop at $t=t+t^{\prime}$ until $t>t_{0}$. The finish of the computational algorithm and the objective functions of the LE-FPL were obtained. The calculation process can be schematised as in Figure 3. 


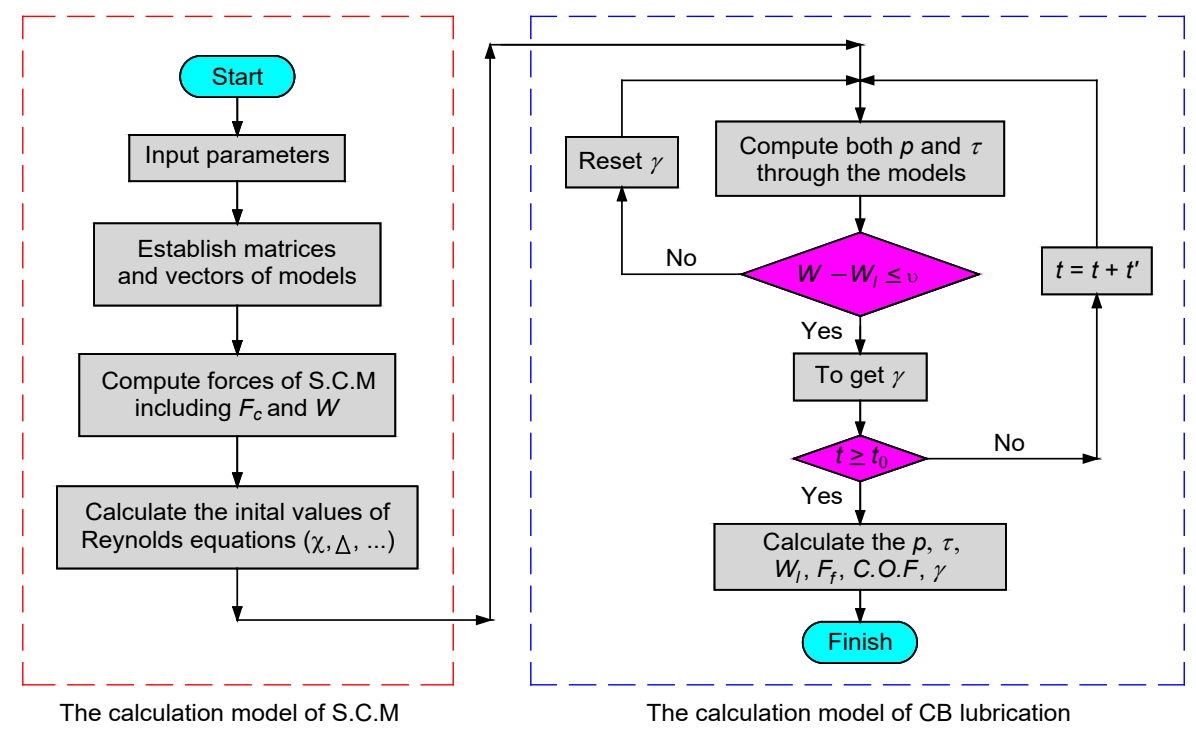

Figure 3. Algorithm flowchart of the computational model.

\section{RESULTS AND DISCUSSION}

Table 3 shows the parameters used in the simulation. The pressure acting on the piston based on the experimental data in reference [17] was applied to calculate the dynamic load $W$ under various crankshaft speeds shown in Figure 4(a). Figure 4(b) sets out the results of the $W$.

Table 3. Parameters of the SCM and the CB

\begin{tabular}{|c|c|c|c|c|c|}
\hline Parameter & Value & Parameter & Value & Parameter & Value \\
\hline$m_{\mathrm{p}}(\mathrm{kg})$ & 0.264 & $l(\mathrm{~m})$ & 0.1295 & $r_{b}(\mathrm{~m})$ & 0.025 \\
\hline$m_{\mathrm{s}}(\mathrm{kg})$ & 0.095 & $r(\mathrm{~m})$ & 0.040 & $\kappa(\mu \mathrm{m})$ & 10 \\
\hline$m_{\mathrm{b}}(\mathrm{kg})$ & 0.250 & $B_{w}(\mathrm{~m})$ & 0.020 & $\eta($ Pas $)$ & 0.02 \\
\hline
\end{tabular}

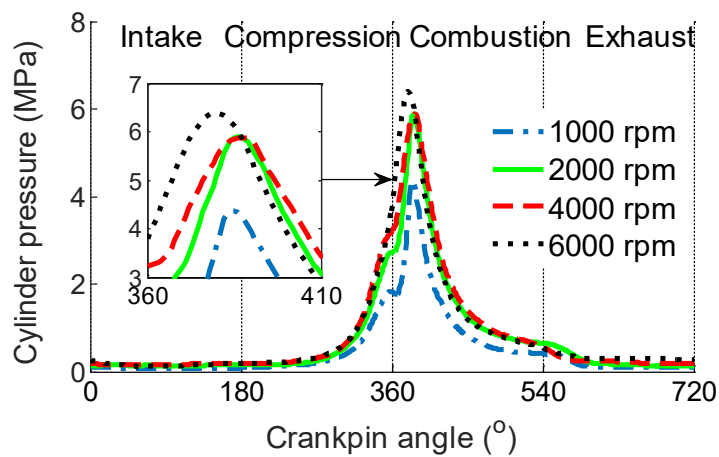

(a)

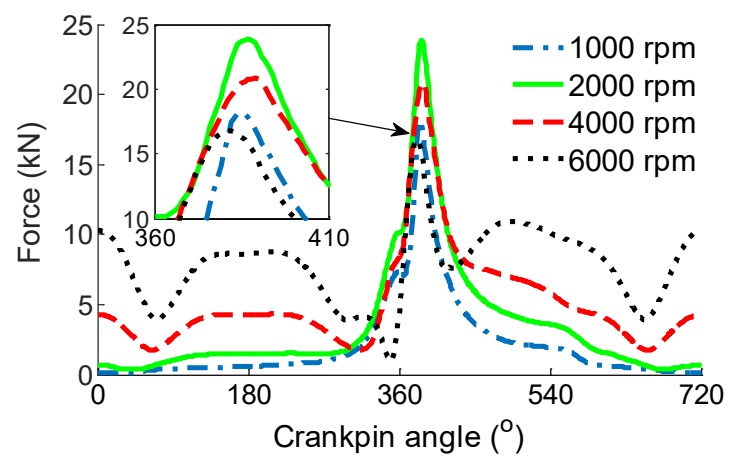

(b)

Figure 4. The impact forces on the CB of (a) combustion gas pressure of experimental data and (b) impacting forces of $W$ on the CB under various angular speeds.

Figure 4(b) shows, in the case of different crankshaft speeds and rotation angles, the impact load continuously varies. At the crankshaft rotation angle from $360^{\circ}$ to $410^{\circ}$ corresponding to the combustion stroke of the engine, the maximum and minimum magnitude of $W$ is obtained at $2000 \mathrm{rpm}$ and $6000 \mathrm{rpm}$, respectively. Still, conversely, outside of the rotation angle of $360^{\circ}$ to $410^{\circ}$, the maximum $W$ is obtained at $6000 \mathrm{rpm}$. This is because of the effect of the centrifugal inertial force of the big-rod-end and the angular speed of the crankshaft in Eq. (8). Thus, the LE-FPL of the CB can be affected by the operation parameters of the CB. To clarify this issue, the influence of the crankshaft speed, bearing radius and width of the $\mathrm{CB}$ was investigated in the following subsections.

\section{Influence of Angular Speed}

The relative speed of the shaft and bearing is given as $u=\omega r_{b}$. Thus, different angular speeds $\omega$ and the corresponding dynamic load $W$ in Figure 4(b) are applied to study the effects on the LE-FPL of the CB. Figure 5 presents the distributions of the oil film pressure and shear stress on the CB surfaces under a dynamic load $W$ at $2000 \mathrm{rpm}$. The results show that the oil film pressure is mainly distributed in a range of $90^{\circ}$ to $180^{\circ}$ of the circumferential coordinate with the bearing width, and its maximum magnitude is $88.8 \mathrm{MPa}$ at $158^{\circ}$ and $B_{w}=0.01 \mathrm{~m}$ of the bearing width. Besides, the shear stress 
is usually evenly distributed along the bearing width. However, in the circumferential coordinate, it varies greatly and reaches the maximum magnitude at $178^{\circ}$.

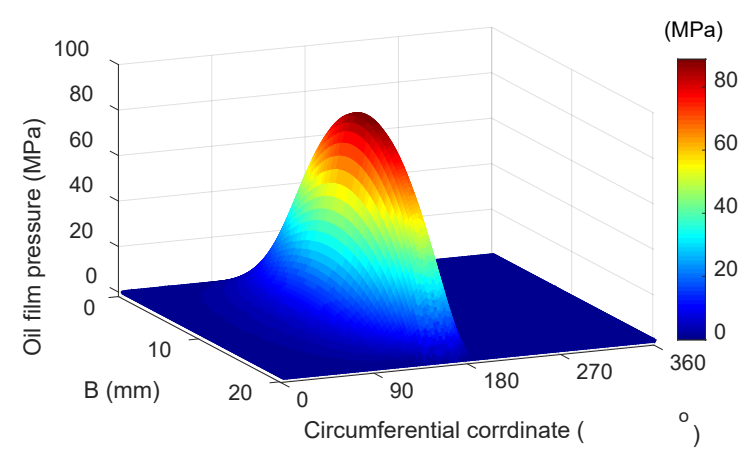

(a)

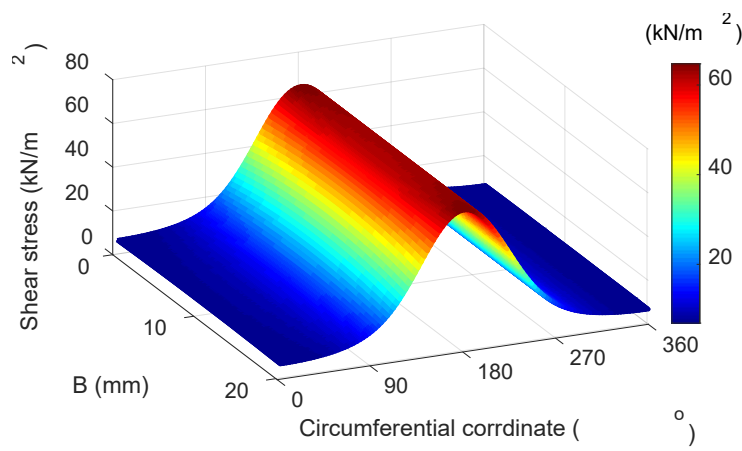

(b)

Figure 5. The results of (a) pressure distribution and (b) shear stress distribution on the CB surface at an angular speed of $2000 \mathrm{rpm}$.

Figure 6 presents the oil film pressure and shear stress distributions at $B_{w}=0.01 \mathrm{~m}$ of the bearing width with various angular speeds. Figure 6(a) shows that the oil film pressure strongly depends on both dynamic load $W$ and angular speed $\omega$. The maximum pressures obtained at $2000 \mathrm{rpm}$ and $6000 \mathrm{rpm}$ are $88.8 \mathrm{MPa}$ and $43.54 \mathrm{Mpa}$, corresponding to the dynamic load $W$, as shown in Figure 4(b). Thus, the maximum pressure at $2000 \mathrm{rpm}$ is greatly increased by $50.96 \%$ compared to the maximum pressure at $6000 \mathrm{rpm}$. In addition, the oil film shear stress mainly depends on the angular speed $\omega$ and the value of shear stress increases with the increase of angular speed and vice versa. In addition, the shear stress mainly depends on and is consistent with the trend of the angular speed.

The CB's load-bearing capacity $W_{l}$ and friction force $F_{f}$ are then calculated on the basis of Figure 6 and plotted in Figures 7(a) and 7(b). It is found that the maximum $W_{l}$ obtained at $2000 \mathrm{rpm}$ and $6000 \mathrm{rpm}$ is $24.84 \mathrm{kN}$ and $16.87 \mathrm{kN}$, and the maximum $W_{l}$ at $2000 \mathrm{rpm}$ is also significantly increased by $32.08 \%$ in comparison with the maximum $W_{l}$ at 6000 $\mathrm{rpm}$. This result is because of increasing of the maximum oil film pressure in Figure 6(a). Besides, the $W_{l}$ are similar to the $W$ in Figure 4(b) under different angular speeds. This is due to the oil film pressure varied to satisfy the condition of $W_{l}$ equilibrium with $W$. Observing Figure 7(b), we also find that the friction force increases sharply with the increase of the angular speed $\omega$. The maximum $F_{f}$ obtained at $2000 \mathrm{rpm}$ and $6000 \mathrm{rpm}$ are $46.38 \mathrm{~N}$ and $113.20 \mathrm{~N}$, and the maximum $F_{f}$ at $2000 \mathrm{rpm}$ is smaller than that of $6000 \mathrm{rpm}$ by $59.03 \%$. Additionally, the friction force reaches its maximum at a high angular speed of $6000 \mathrm{rpm}$, and this means the friction force acting on the CB is the greatest at this point. This is because of the increase of the relative velocity, which leads to an increase in the shear stress and frictional resistance of the oil film.

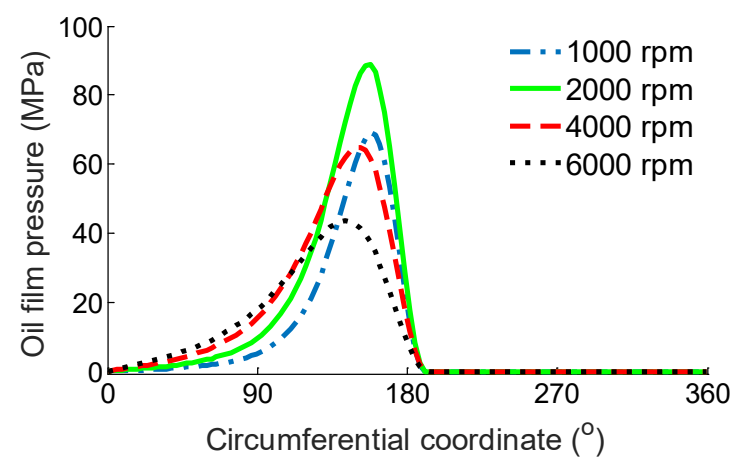

(a)

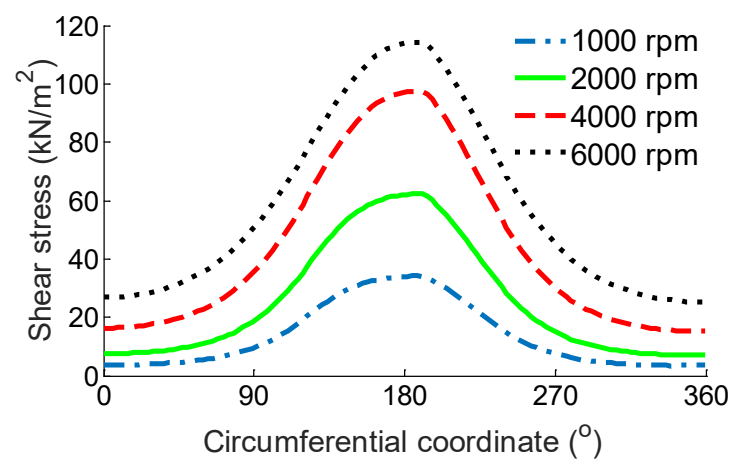

(b)

Figure 6. (a) Pressure distribution and (b) shear stress distribution at $B_{w}=0.01 \mathrm{~m}$ of the bearing width under different angular speeds.

As shown in Figure 7(c), the friction coefficient changes with the variation of $W_{l}$ and $F_{f}$ and decreases with increasing angular speed $\omega$. The analysis shows that in the combustion stroke of the engine (that is, the crankpin angle is in $360^{\circ} \sim$ $410^{\circ}$ ), the maximum dynamic load $W$ affects not only the lubrication efficiency of $\mathrm{CB}$, but also the engine power. Especially the dynamic load $W$ reaches the maximum when the speed is maintained at $2000 \mathrm{rpm}$, while the friction force $F_{f}$ and COF are relatively small. This is consistent with actual engineering experience $[1,5]$.

In Figure 7(d), the influence of eccentricity ratio $\gamma$ on CB stability at different angular speeds is also studied. The results indicate that increasing angular speed can reduce the eccentricity ratio and vice versa. The eccentricity ratio reaches its maximum at a low speed of $1000 \mathrm{rpm}$ and its minimum is at a high speed of $6000 \mathrm{rpm}$. Therefore, the eccentricity ratio also affects the lubrication efficiency and stability of the journal. Especially when the rotation speed is at $6000 \mathrm{rpm}$, 
the eccentricity ratio changes less, indicating the lubrication capacity and rotation of the journal are more stable at this time.

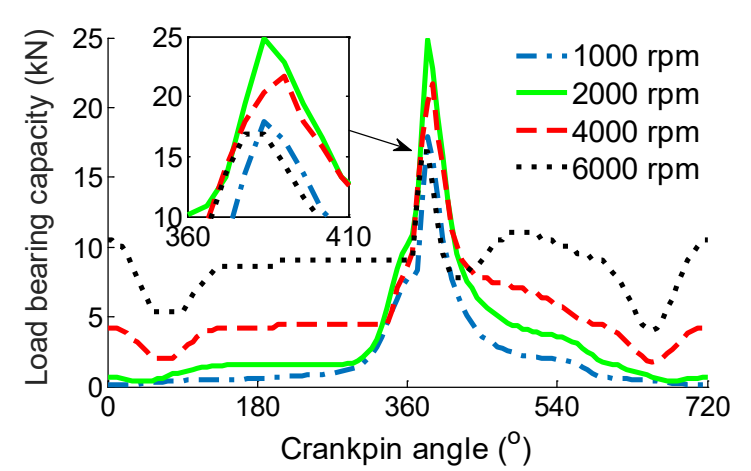

(a)

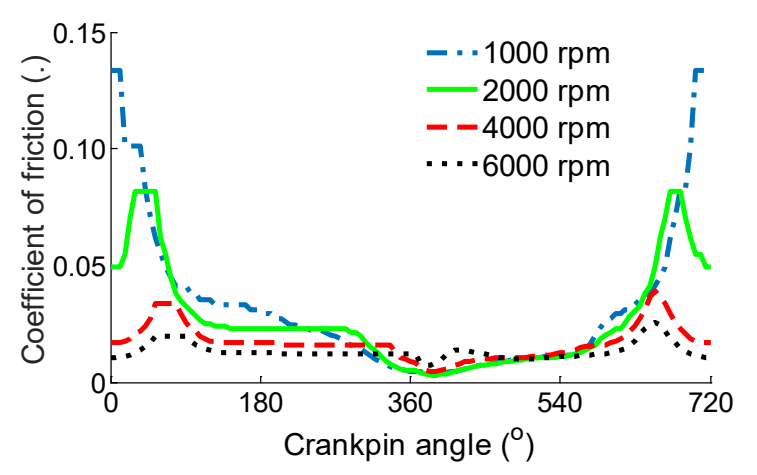

(c)

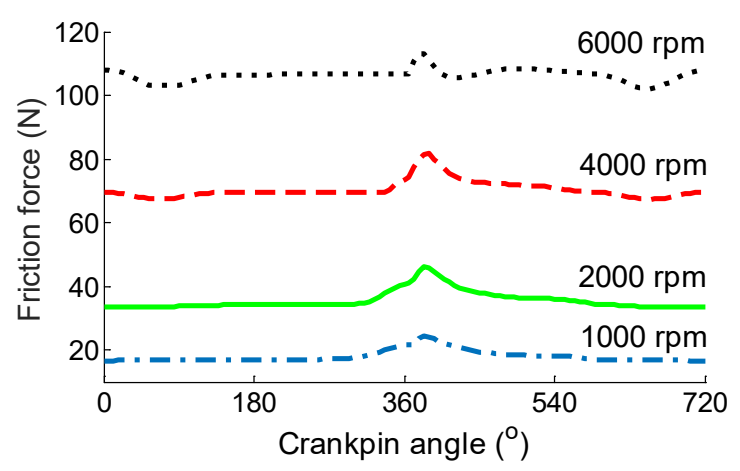

(b)

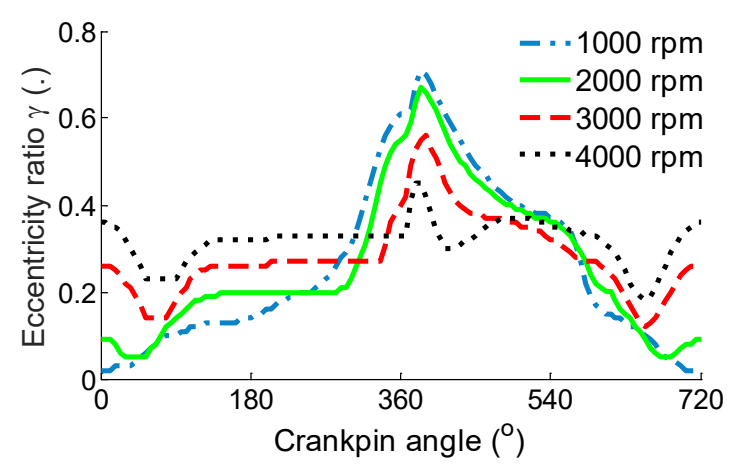

(d)

Figure 7. Influence of angular speeds on the LE-FPL with (a) CB's load-bearing capacity, (b) friction force, (c) friction coefficient, and (d) eccentricity ratio between journal and bearing.

\section{Influence of Bearing Radius}

In this part, the influence of bearing radius and width on LE-FPL under the maximum dynamic load $W$ of the engine at 2000 speed was studied. Figure 8(a) and 8(b) respectively present the distribution of the oil film pressure and shear stress at $B_{w}=0.01 \mathrm{~m}$ of the bearing width with bearing radius of $r_{b}=\{0.015,0.020,0.025,0.030,0.035\} \mathrm{m}$. It included that the oil film pressure decreases and the shear stress increase when the bearing radius gradually increases from 0.015 $\mathrm{m}$ to $0.035 \mathrm{~m}$. Additionally, the $W_{l}$ and $F_{f}$ with different bearing radius are shown in Figure 9(a) and 9(b), respectively. It observed that the change of radius almost no influence on the load force $W_{l}$ in comparison with the dynamic load $W$ under the condition of $W_{l}$ equilibrium with $W$ as illustrated in Figure 9(a). The reason for this result is that the calculation area increases due to the rise in bearing radius leading decrease of oil film pressure shown in Figure 8(a) to stabilise the load $W_{l}$ and vice versa. This also verifies the accuracy of the mathematical model and algorithm program.

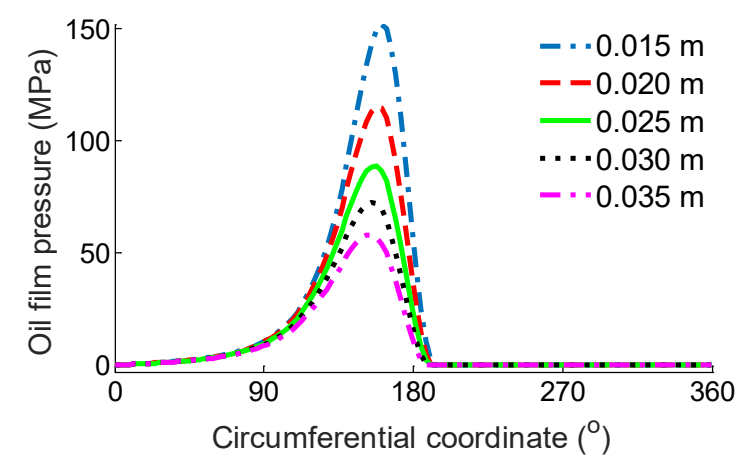

(a)

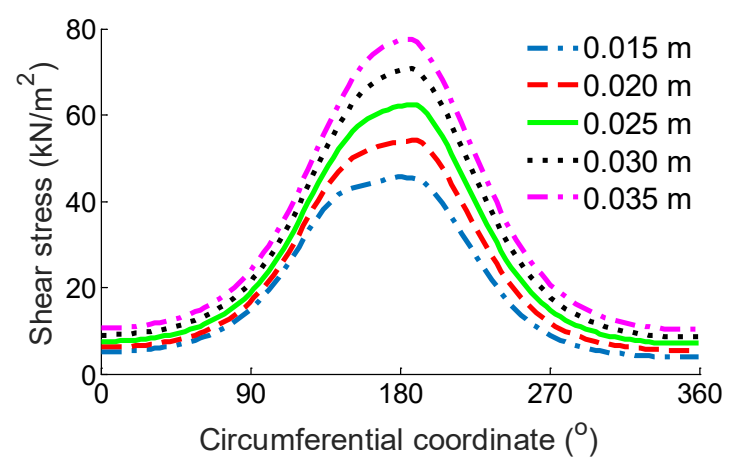

(b)

Figure 8. The results of (a) pressure distribution and (b) shear stress distribution at $B_{w}=0.01 \mathrm{~m}$ of the bearing width with different bearing radius.

As indicated in Figure 9(b), the amplitudes of friction force $F_{f}$ tend to increase with the increase of the bearing radius $r_{b}$. It is attributed to the rise of the relative speed of oil film $\left(u=\omega r_{b}\right)$ and the calculation area $A_{b}$ affecting the oil film shear stress and friction resistance. Because the load $W_{l}$ is not varied with the different bearing radius, the COF will 
increase due to the increase of the CB's friction force, as shown in Figure 9(c). Moreover, seen from Figure 9(d), bearing radius has little effect on eccentricity ratio, and the gap of the $\mathrm{CB}$ shows a pronounced tendency to decrease with the radius increases.

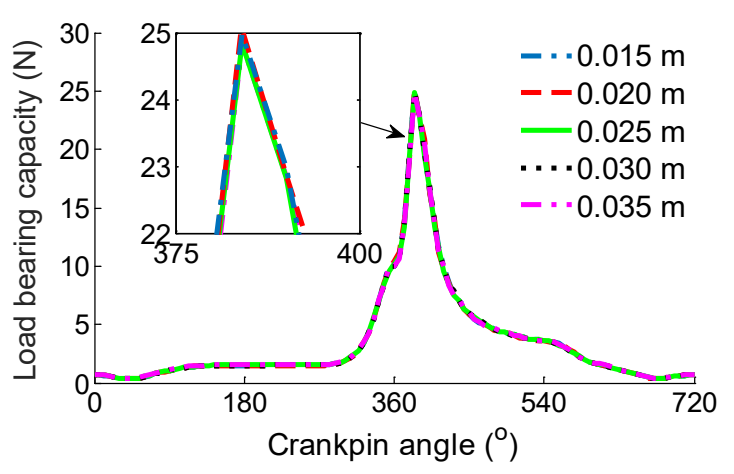

(a)

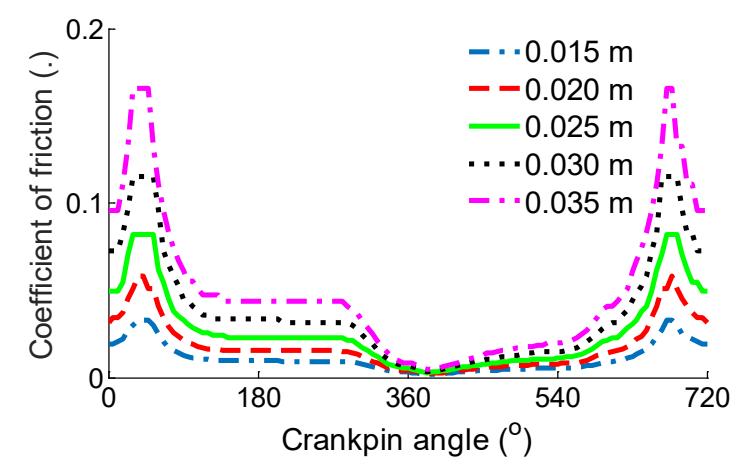

(c)

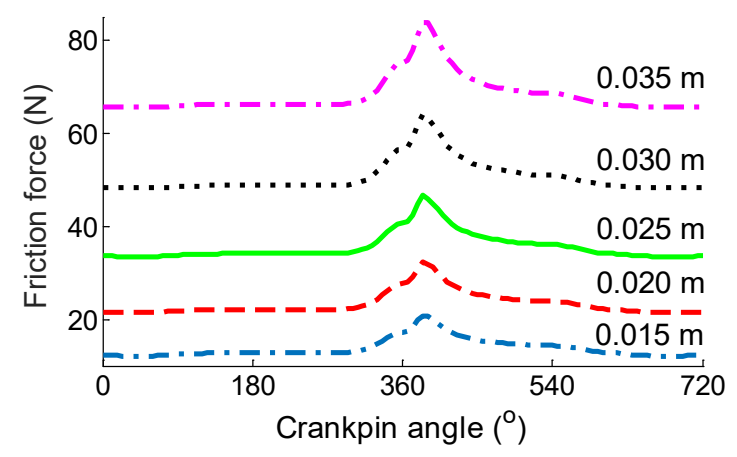

(b)

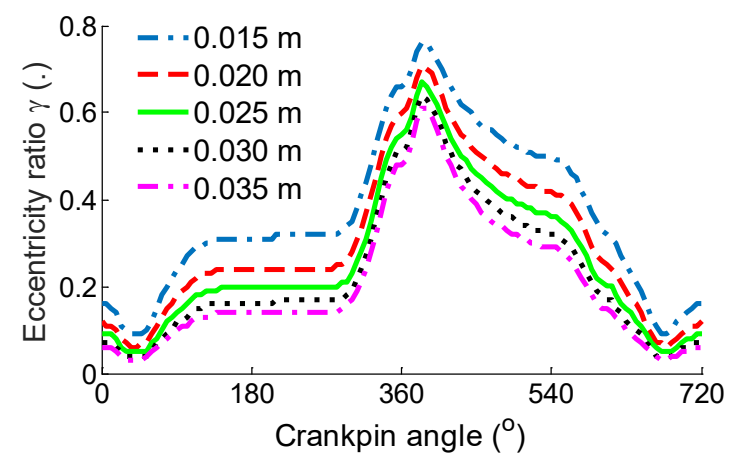

(d)

Figure 9. Influence of the bearing radius on the LE-FPL with (a) CB's load-bearing capacity, (b) friction force, (c) friction coefficient, and (d) eccentricity ratio between journal and bearing.

\section{Influence of Bearing Width}

The influence of the bearing width $B_{w}=\{0.010,0.015,0.020,0.025,0.030\} \mathrm{m}$ on the LE-FPL with the same $W$ at $2000 \mathrm{rpm}$ is demonstrated in Figure 10. As Figure 10(a) shows, the load $W_{l}$ generated from the oil film pressure does not change significantly in comparison with $W$ when the bearing width gradually increases from $10 \mathrm{~mm}$ to $30 \mathrm{~mm}$. This is because the oil film pressure changes to stabilise the load $W_{l}$. In addition, $F_{f}$ and COF are going to increase, as seen in Figure 10(b) and 10(c), which will directly cause greater friction power loss. However, in Figure 10(d), the width variation has little effect on the eccentric ratio between the journal and bearing.

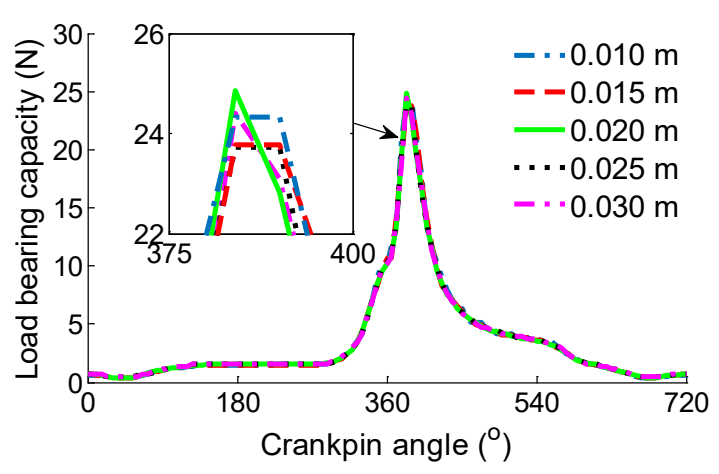

(a)

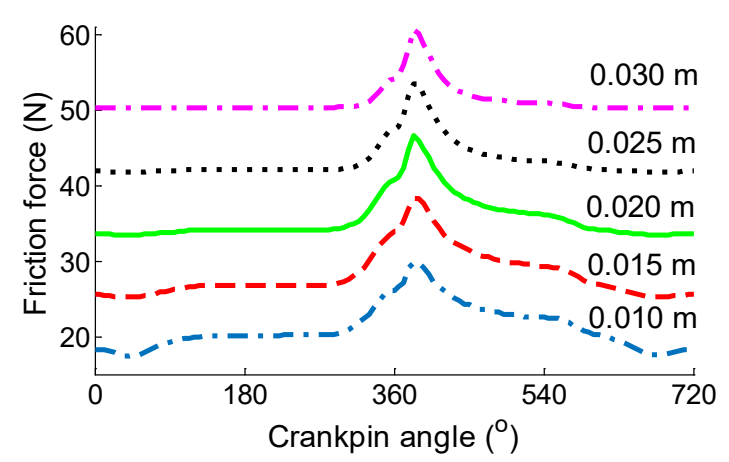

(b) 


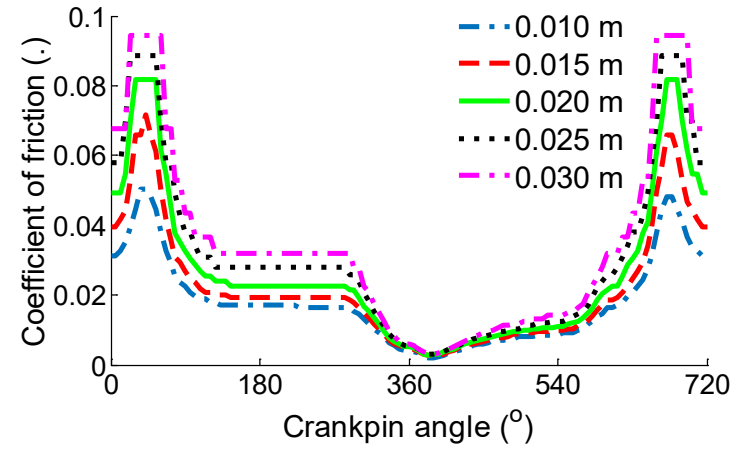

(c)

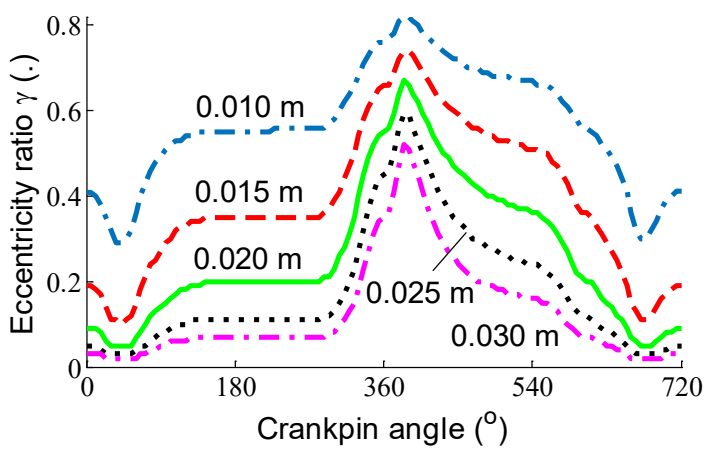

(d)

Figure 10. Influence of the bearing widths on the LE-FPL with (a) CB's load-bearing capacity, (b) friction force, (c) friction coefficient, and $(\mathrm{d})$ eccentricity ratio between journal and bearing.

\section{CONCLUSION}

In this study, a new numerical approach combined with the SCM dynamics model and the CB lubrication model was proposed to study the LE-FPL of engines, and the results are as follows.

i. Increasing the angular speed of the engine can not only effectively reduce the eccentricity ratio of the CB but also increase the minimum oil film thickness of the $\mathrm{CB}$, which will directly enhance the CB's lubrication efficiency. However, the friction force will also increase, especially the friction force increases by $59.03 \%$ at $6000 \mathrm{rpm}$ compared with that of $2000 \mathrm{rpm}$, which increases the friction power loss of engines. The research result shows that the LE-FPL of engines could be better improved at an angular speed of $2000 \mathrm{rpm}$.

ii. Increasing radius or width for the $\mathrm{CB}$ could help reduce the eccentricity ratio $\gamma$, thus improving the lubrication efficiency. Additionally, the CB's dimensional change has a great influence on the friction force and friction coefficient, thereby increasing the friction power loss of engines.

iii. Improving the friction properties and reducing the friction power loss are two conflicting issues. On the one hand, this study enriches the knowledge system of the LE-FPL of engines. On the other hand, it also provides an essential reference for the optimisation design parameters of the SCM.

\section{ACKNOWLEDGEMENT}

This work is supported by the Open Fund Project of Hubei Key Laboratory of Intelligent Transportation Technology and Device, Hubei Polytechnic University, China (No. 2021XZ107, 2020XZ107), and by the Key Project of Scientific Research Plan of Education Department of Hubei Province (No. D20204501) and of Hubei Polytechnic University (No.21xjz02A).

\section{REFERENCES}

[1] Y. Lia et al., "Dynamic analysis and optimisation design of a planar slider-crank mechanism with flexible components and two clearance joints," Mech Mach Theory, vol. 99, no. May, pp. 37-57, 2016, doi: 10.1016/j.mechmachtheory.2015.11.018.

[2] X. Meng, L. Ning, Y. Xie, and V. Wong, "Effects of the connecting-rod-related design parameters on the piston dynamics and the skirt-liner lubrication," Proc. Inst. Mech. Eng. D, vol. 227, no. 6, pp. 885-898, 2013, doi: 10.1177/0954407012464025.

[3] B. Zhao, X. Dai, Z. Zhang, and Y. Xie, "A new numerical method for piston dynamics and lubrication analysis," Tribol. Int., vol. 94, no. February, pp. 395-408, 2016, doi: 10.1016/j.triboint.2015.09.037.

[4] S. Cho, S. Ahn, and Y. Kim, "A simple model to estimate the impact force induced by piston slap," J. Sound Vib., vol. 255, no. 2, pp. 229-242, 2002, doi: 10.1006/jsvi.2001.4152.

[5] Guzzomi, D. Hesterman, and B. Stone, "Variable inertia effects of an engine including piston friction and a crank or gudgeon pin offset," Proc. Inst. Mech. Eng. D, vo. 222, no. 3, pp. 397-414, 2008, doi: 10.1243/09544070JAUTO590.

[6] S. Mansouri, and V. Wong, "Effects of piston design parameters on piston secondary motion and skirt-liner friction," Proc. Inst. Mech. Eng. J, vol. 219, no. 6, pp. 435-449, 2005, doi: 10.1243/135065005X34026.

[7] N. Aslfattahi et al., "MXene based new class of silicone oil nanofluids for the performance improvement of concentrated photovoltaic thermal collector," Sol. Energy Mater. Sol. Cells, vol. 211, pp. 110526, 2020, doi:10.1016/j.solmat.2020.110526.

[8] F. Rubbi et al., "Performance optimisation of a hybrid PV/T solar system using Soybean oil/MXene nanofluids as a new class of heat transfer fluids," Sol. Energy, vol. 208, pp. 124-138, 2020, doi:10.1016/j.solener.2020.07.060.

[9] L. Samylingam et al., "Thermal and energy performance improvement of hybrid PV/T system by using olein palm oil with MXene as a new class of heat transfer fluid," Sol. Energy Mater. Sol. Cells, vol. 218, pp. 110754, 2020, doi:10.1016/j.solmat.2020.110754.

[10] N. Parashar, N. Aslfattahi, S. Yahya, and R. Saidur, "An artificial neural network approach for the prediction of dynamic viscosity of MXene-palm oil nanofluid using experimental data," J. Therm. Anal. Calorim., pp. 1-12, 2020, doi:10.1007/s10973-020-09638-3. 
[11] B. Gregory, and L. Katia, "Analysis of the dynamics of a slider-crank mechanism with hydrodynamic lubrication in the connecting rod-slider joint clearance," Mech Mach Theory, vol. 46, no. 10, pp. 1434-1452, 2011, doi: 10.1016/j.mechmachtheory.2011.05.007.

[12] X. Wang, J. Zhang, and H. Dong, "Analysis of bearing lubrication under dynamic loading considering micropolar and cavitating effects,” Tribol. Int., vol. 44, no. 9, pp. 1071-1075, 2011, doi: 10.1016/j.triboint.2011.05.002.

[13] R. Jiao, N. Van, Q. Le, and C. Bui, "Optimal design of micro-dimples on crankpin bearing surface for ameliorating engine's lubrication and friction," Ind. Lubr. Tribol., vol. 73, no. 1, pp. 52-59, 2020, doi:10.1108/ILT-04-2020-0152.

[14] H. Zhang et al., "Boundary slip surface design for high speed water lubricated," Tribol. Int., vol. 79, no. November, pp. 3214, 2014, doi: 10.1016/j.triboint.2014.05.022.

[15] Z. Wu et al., "Design and optimisation of textures on the surface of crankpin bearing to improve lubrication efficiency and friction power loss (LE-FPL) of engine," Proc. Inst. Mech. Eng. J, vol. 235, no. 6, pp. 1139-1149, 2020, doi:10.1177/1350650120942009.

[16] V. Nguyen, Z. Wu, and V. Le, "Optimisation of crankpin bearing lubrication under dynamic loading considering effect of micro asperity contact," Ind. Lubr. Tribol., vol. 72, no. 10, pp. 1173-1179, 2020, doi: 10.1108/ILT-02-2020-0072.

[17] B. Zhao et al., "Modeling and analysis of planar multibody system with mixed lubricated revolute joint," Tribol. Int., vol. 98, no. March, pp. 229-241, 2016, doi: 10.1016/j.triboint.2016.02.024.

[18] K. Christian et al., "Investigation of journal orbit and flow pattern in a dynamically loaded journal bearing," Tribol. Int., vol. 114, no. October, pp. 450-457, 2017, doi: 10.1016/j.triboint.2017.04.013.

[19] M. Braun, and W. Hannon, "Cavitation formation and modelling for fluid film bearings: a review," Proc. Inst. Mech. Eng. $J$, vol. 224, no. 9, pp. 839-863, 2010, doi: 10.1243/13506501JET772.

[20] Z. Wu, V. Nguyen, Z. Zhang, and L. Zheng, "Study on curved surface design of sliding pair based on stepped topography model," Ind. Lubr. Tribol., vol. 72, no. 1, pp. 86-92, 2019, doi: 10.1108/ILT-04-2019-0121. 